\title{
Design of Experiments for the optimization the process parameters of thixotropic aluminum alloy
}

\author{
A. Barcellona $a^{1, a}$, L. Fratini ${ }^{1, b}$ and D. Palmeri ${ }^{1, c}$ \\ ${ }^{1}$ Department of Tecnologia Meccanica, Produzione ed Ingegneria Gestionale, University of \\ Palermo, Viale delle Scienze, Palermo, 90128, Italy \\ abarce@dtpm.unipa.it, babaqus@dtpm.unipa.it, 'palmeri@dtpm.unipa.it
}

Keywords: Thixoforming, Semisolid State, Design of Experiments, Central Composite Design.

\begin{abstract}
The success of the thixoforming process depends on the possibility to confer to material, when it is found in the semisolid state, a microstructure characterized by globular particles of solid phase surrounded by a continuous film of liquid phase; such microstructure is obtainable through particular thermo-mechanical treatments. In the present research, in order to optimize the influence of process parameters in the step in which the thixotropic properties are conferred to the AA7075 aluminum alloy, the statistic technique of the Design Of Experiments (DOE) has been used. The advantages in the application of such technique are expressible in terms of reduction the times of development of process and more efficient use of resources. A Central Composite Design factorial plan with two levels has been realized to allow the evaluation of experimental error and to check the adequacy of the model. The experimental tests foreseen from the same plan have been therefore performed. Using the method of the response surfaces (RSM), the function of response has been formulated, and the analysis of the experimental data has been realized by the linear regression method. Finally, the analysis of variance (ANOVA) allowed to value the causes of variability of the results. Statistic test has been performed on the significance of single factors and their interactions.
\end{abstract}

\section{Introduction}

Thixoforming process consists in the forming of material when it is in its semisolid state, i.e. at a temperature between solidus and liquidus ones. In these conditions it is possible to combine the fluidity of the liquid state to the formability in the solid state, so combining both advantages of the conventional foundry and forging techniques. Typical solidification structure of aluminum alloys in equilibrium conditions is a dendritic type, that shows low formability. When a spheroidal microstructure is existing, formability and final mechanical characteristics grow. In particular, when the material is in the semisolid state, it acquires "thixotropic" properties. In presence of liquid phase, around the spheroidal grains, during a deformation process, a drastic decreasing of interactions and friction between grains occurs. The mechanical agitation, executed to dendritic crushing, has been performed by employing a crank mechanism and an electric engine. By means of this agitation, a shear action that allows to obtain the globular structure, has been conferred to the material. After the first treatment, each specimen has been successively subjected to a re-heating treatment. This treatment was executed in three step in order to be sure about the temperature control. Cooling has been conducted with high cooling rate, in order to freeze the obtained structures at environment temperature [1], [2]. In this research the experiments performed in order to determine the best set of input parameters that ensure the optimization of responded parameters, have been determined by means of the statistical technique of design of experiments (DOE), [3]. The experimentation empirically aimed to widen and to deepen the knowledge on the process. One of the main aim of the experimentation work is the resetting, or at least the pull down, of effects induced from the variability. Another aim, equally important, consists in carrying the process to work in optimal conditions. A planned experiment allows to know in detail the background in which the process was realized and to create conditions of congruence between input factors of process and the output factors, in order to identify the more proper values to assign to each influence factor. With the design of experiments it is 
possible to define the criteria and the rules to be followed during the experimentation and also the principles and the modalities to adopt in the analysis and in the elaboration of the obtained data. The experimentation activity must be conducted according to procedures that allow to overcome the improvisation and the approximation that are connected to the experimentation carried out with the classic method, according to which the different influence factors, that are present, have made to vary one to the time, maintaining the others to a fixed value of reference. In facts it has been demonstrated that the simultaneous variation of the levels of influence factors, according to a proper design, is an essential requirement in order to reduce the costs of tests and to improve the validity of results.

\section{Planning of DOE}

The design of experiments starts from a careful overview of process with the aim to enunciate a theory that has to be verified by the analysis of the outputs of the experimental tests. In previous studies the thermo-mechanical treatment, in order to confer the thixotropic properties, has been set up in two different steps. In the first step, the time of mechanical treatment has been chosen as "influential controllable factor"; in the second step, so called re-heating, the time of permanence of the semisolid temperature has been chosen as "influential factor". The output of process has been valued through microstructural analysis, as shown in Fig. 1, in terms of mean grain dimension, shape factor and micro-hardness Vickers tests. The goals of the experimentation may be summarized in the following points:

-To determine which controllable factors, so called $\mathrm{X}$, are more influential on the so called $\mathrm{Y}$ answer;

- To determine the significant factors at the level that ensure an Y answer near to a target value;

- To determine the significant factors at the level that ensure minimal variability of Y;

- To determine the significant factors at the level that ensure the minimal sensibility of $Y$ in the comparisons of the variability due to the not controllable factors.

For each factor it is used a levels coding, i.e., for each factor $\mathrm{X}$ there are two levels $\xi_{i}$, with $i=1,2$. For factors related to continuous variables, as in this case, the following transformation has been used:

$$
\xi_{i}=2 \cdot \frac{X_{i}-\bar{X}}{\Delta X}
$$

At the low level it is usually coupled the conventional number -1 and to the high level the number 1 . Before the planning of the experiments, it is proper to define all the necessary operations for the performance of each treatment. Every treatment has been executed bringing the AA 7075 specimen to the temperature of $950^{\circ} \mathrm{C}$. Subsequently each specimen has been cooled in oven until the semi-solid temperatures of $610{ }^{\circ} \mathrm{C}$. The subsequent treatment of mechanical agitation has been executed with different durations for each treatment. After that, in order to generate the necessary conditions of metastability for the subsequent sharpening of structure in the re-heating step, a water cooling down to room temperature has been executed. Finally, in order to obtain an uniform temperature in the specimen, the treatment of re-heating has been executed in three different steps.
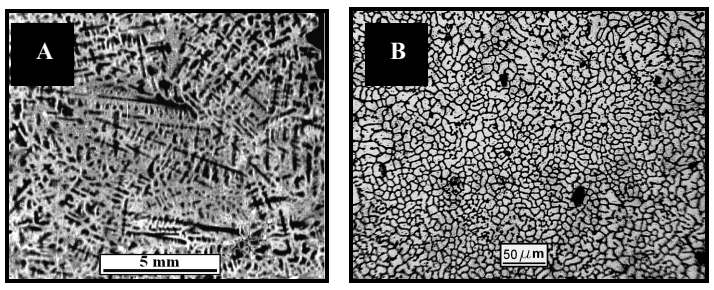

Fig. 1: A - typical solidification dendritic structure of AA 7075, B - globular thixotropic structure obtained after the thermo-mechanical treatment.

The third step has been executed at the temperature of $610^{\circ} \mathrm{C}$ and each specimen has been maintained at such temperature for a different time for each test. The aim of this research is therefore the 
determination of the time of mechanical agitation and the permanence time in the third step of the re-heating treatment that concur to obtain a homogeneous fine and uniform globular structure. The time of mechanical agitation $\mathrm{X}_{1}$, and the permanence time at $610{ }^{\circ} \mathrm{C}$ in the last step of the re-heating treatment $\mathrm{X}_{2}$, are considered as measurable and controllable factors, because they have influence on the answer variables. The values of the mean grain dimension, shape factor and Vickers micro-hardness are the employed parameters in order to characterise the microstructure of thixotropic alloy. Therefore these parameters are the output variables of plan of the experiments. The factorial design is an effective tool in order to analyse the single and the combined influence of the influential factors of process on the experimental responses, because it concurs the esteem of the main effects of the factors and their interactions. In the explorative phase of process a factorial design, with two factors and two levels $\left(2^{2}\right)$ with some point to the centre, like copy, for having one greater precision in the esteem of the mean effects, has been used. The sample space is therefore a square. In order to deepen the analysis, the Response Surface Method (RSM) has been used. The first objective is the determination of the maximum (or the minimum) of the response surface and therefore of the corresponding input factors, the second objective is the formulation of the response function. Firstly the time levels of mechanical agitation $\left(\mathrm{x}_{1}\right)$ and permanence time at $610^{\circ} \mathrm{C}$ in the last step of the re-heating treatment $\left(\mathrm{x}_{2}\right)$, that maximise the response $(\mathrm{y})$ of thixotropic treatment, must be determined. The $\mathrm{y}$ response is a $\left(\mathrm{x}_{1}, \mathrm{x}_{2}\right)$ function in the form:

$$
y=f\left(x_{1}, x_{2}\right)+\varepsilon
$$

where $\varepsilon$ is the error observed in the response $y$. If the response is indicated with $\eta=f\left(x_{1}, x_{2}\right)$, the surface that it represents is called response surface. Firstly it is necessary to find an opportune approximation of the actual function $\mathrm{y}=\mathrm{f}\left(\mathrm{x}_{1}, \mathrm{x}_{2}\right)$; initially the operating conditions for the process are distant from the actual optimum and in this case it has been used a linear models as approximation of the true surface in this region of the $\mathrm{x}$. The model of the first order function is expressed as follow:

$$
y=\beta_{0}+\beta_{1} x_{1}+\beta_{2} x_{2}+\ldots+\beta_{k} x_{k}+\varepsilon
$$

The experiments along the distance of faster climb were realized until increments in the response were observed. At this point a new model of the first order is used, and the experiments are executed along a new route of faster climb. This way is followed until a lack of adaptation of the model of the first order is observed and at this point the process is near of the optimum condition. When the optimal is near, it has been employed a polynomials function of higher order as a second order:

$$
y=\beta_{0}+\sum_{i=1}^{k} \beta_{i} x_{i}+\sum_{i=1}^{k} \beta_{i i} x_{i}^{2}+\ldots+\sum_{i<j} \sum \beta_{i j} x_{i} x_{j}+\varepsilon
$$

In order to analyze the data of experiments the method of the linear regression has been employed. The regression model adapted in matrix shape is:

$$
\hat{y}=X \hat{\beta}
$$

The difference between the actual observations $y_{\mathrm{i}}$ and the correspondents adapted values $\hat{y}_{i}$ is the residual:

$$
e_{i}=y_{i}-\hat{y}_{i}
$$

The study of the residual concurs to discover the eventual inadequacy of the model. If the model is well adapted the residual does not show a diagram with evident bonds among any variables. The regression has been defined as one function that interpolates at best the experimental point. Therefore it is necessary to have a measure of the goodness of such interpolation. The analysis of the variance of results (ANOVA) has been executed in order to establish if the variability of the results is due to the case or to the applied effect by the levels. By means of statistical tests some indications on the significance of the factors and their interactions have been obtained. The employed design of experiments is the central composite design (CCD) and it was used in order to adapt a second order model. It has the characteristic to estimate the factors of the first order and of the second mixed order 
in an independent way between them; it also estimate those of second order, with one light dependency between them. The determined design of experiments is an increased factorial design $2^{2}$ with three center points, as it is shown in table.

Tab. 1: DOE

\begin{tabular}{|c|c|c|c|c|}
\hline Test $\mathrm{n}^{\circ}$ & $\mathrm{A}$ & $\mathrm{B}$ & $\begin{array}{c}\text { Time of mechanical } \\
\text { treatment (min) }\end{array}$ & $\begin{array}{c}\text { Permanence time in the third step of } \\
\text { re-heating treatment (min) }\end{array}$ \\
\hline 1 & -1 & 1 & 15 & 30 \\
\hline 2 & 1 & -1 & 25 & 30 \\
\hline 3 & -1 & 1 & 15 & 20 \\
\hline 4 & 1 & -1 & 25 & 20 \\
\hline 5 & 0 & 0 & 20 & 25 \\
\hline 6 & 0 & 0 & 20 & 25 \\
\hline 7 & 0 & 0 & 20 & 25 \\
\hline
\end{tabular}

\section{Experimental Results}

The tests $n^{\circ} 1$ and 3, even if subjected to the same treatment of mechanical agitation, have introduced similar responses (nearly identical) for the mean grain dimension, but the values of micro-hardness and of shape factor are strongly different. The same situation it has been found also for tests $\mathrm{n}^{\circ} 2$ and 4, but in this case the diversities are mainly marked; in facts the three responses, micro-hardness, mean grain dimension, and shape factor, are different. Also for the three central tests $n^{\circ} 5,6$ and 7 , the obtained results have been various. A microstructure with better mean grain dimension has been observed in the three central tests, while the Vickers micro-hardness values have not been the best ones. The shape factor observed after the re-heating treatment is similar for all the conduced tests; therefore, one optimal structure, in absolute, it has not been obtained. The attainment of responses strongly various between them in the tests realized with the same levels of the factors has been attributed to the impossibility to control of the noise factors not taken into account, such as the precision of the cooling rate of specimen. The analysis of the influence of the mean grain dimension has produced a response in which the p-value is small. The value of the coefficient of multiple determination $\mathrm{R}^{2}$ is high, and also the one of the statistic $R_{a d j}^{2}$. The regression model for the mean grain dimension is therefore good. The same result is not evidenced for the values of micro-hardness and shape factor. It can be thought that the dispersion of the responses is due to the choice of the values assigned to the levels of the controlled factors, that are very near between them; in facts the compromise between the two bonds wide excursion and operating practicability is fundamental in the choice of the levels. The successive step of this research therefore will be to make vary the factors level within a wider interval, starting however from the center-points of this study.

\section{References}

[1] A. Barcellona, D. Palmeri, R. Riccobono: Thermo-Mechanical Treatments On Aluminum Alloys For Microstructure Improvements, $18^{\text {th }}$ International Conference on Production Research (2005)

[2] A.Barcellona, L.Fratini, D.Palmeri: Influence Of Process Parameters For Thixotropic Alloys, AMST'05 (2005)

[3] C.Douglas Montgomery: Design and Analysis of Experiments (1997) 